\title{
Modeling and analysis of the spectral response for AIGaAs/GaAs HPTs for short wavelength optical communication
}

\author{
Hassan A. Khan, ${ }^{1, a)}$ Ali A. Rezazadeh, ${ }^{2}$ and Sarmad Sohaib, ${ }^{3}$ \\ ${ }^{1}$ Department of Electrical Engineering, Lahore University of Management Sciences, Lahore Cantt, \\ 54792, Pakistan \\ ${ }^{2}$ Microwave and Communication Systems Research Group, The University of Manchester, \\ M60 1QD United Kingdom \\ ${ }^{3}$ Department of Electrical Engineering, University of Engineering and Technology, 47080 Taxila, Pakistan
}

(Received 19 January 2011; accepted 2 April 2011; published online 20 May 2011)

\begin{abstract}
Detailed spectral response (SR) modeling for heterojunction bipolar phototransistors (HPTs) is presented in this work. All the related physical parameters are taken into account for the resolution of photogenerated excess minority carrier continuity equations in the active layers of the HPT. The layer dependence of the optical flux absorption profile at near-bandgap wavelengths is also investigated and its generalization as a single-exponential has been refuted for HPTs based on GaAs material systems (InGaP-GaAs/AlGaAs-GaAs). The variation in the responsivity of the device with changing base width is analyzed at various wavelengths and a detailed experimental setup for optical characterization of HPTs is also provided. The measured results at 635, 780, 808, and $850 \mathrm{~nm}$ show good agreement to the modeled data, validating the newly developed theoretical model. (C) 2011 American Institute of Physics. [doi:10.1063/1.3585846]
\end{abstract}

\section{INTRODUCTION}

A renewed interest in heterojunction phototransistors (HPTs) has been seen in the past decade due to their use in the front-end of optoelectronic monolithic microwave/millimeter-wave integrated circuit (OEMMIC) optical receivers based on heterojunction bipolar transistors (HBTs). ${ }^{1,2}$ Monolithic integration implies that the detectors (HPTs/photodiodes) and amplifiers (HBTs) share common epitaxial layers or in some cases device layers are grown on the top of each other. ${ }^{3}$ The latter process is cost ineffective which makes it unattractive for industrial manufacture of photoreceivers. In the shared epitaxial structures, the fabrication compatibility of HPTs and HBTs make them highly attractive in the manufacture of high-speed photoreceivers. However, the inevitable design tradeoffs in this process degrade the photoreceiver performance. ${ }^{4}$ Hence, there is a need to critically analyze several important parameters of these devices which can further revolutionize their use and extend their range of functionality from ordinary sensors to high speed optical networks.

To date, most optical receiver designs have employed pin photodiodes as photodetectors. However, these devices exhibit no internal/intrinsic gain and the increased capacitance due to large intrinsic absorption layer is known to be the limiting factor for high frequency operation of the photoreceiver. ${ }^{5}$ Metal-semiconductor-metal (MSM), on the other hand, have low capacitance due to Schottky-like contact which make them suitable for high frequency operation but their temperature instability, lack of internal gain and complicated epitaxial growth and fabrication process make their use limited for long wavelength optical receivers. ${ }^{6}$ The advantage of HPTs over avalanche photodiodes (APDs) is evident from its low noise operation along with the simple

${ }^{\text {a)} E l e c t r o n i c ~ m a i l: ~ H a s a n . a b b a s . k h a n @ g m a i l . c o m . ~}$ biasing conditions compared to APDs. $^{7}$ It is therefore expected that HPTs provide an exciting alternative to the above mentioned devices for the manufacture of high speed optical receiver for both short and long wavelength communication based on HBTs.

A key performance parameter of detectors is their spectral response (SR). Modeling of SR of photodiodes is extensively reported. $^{8-14}$ Bouhdada et al. ${ }^{8}$ modeled the responsivity of $p-i-n$ photodiodes with the impact of surface recombination velocity. Computer simulation for $\mathrm{Si} p-i-n$ photodiodes is developed and explored by Cappellitte et al. ${ }^{9,10}$, and Hanzaz et al. ${ }^{11}$ performed the same task for $\mathrm{Al}_{x} \mathrm{Ga}_{1-x} \mathrm{~N} p-n$ junction photodiodes and also discussed the influence of doping on the exposed zone thickness. A spectral response model for Schottky photodiodes has also been reported by Zheng et al. ${ }^{12}$ and the temperature dependent model for infrared photodiodes has been analyzed by Gonzales-Cuevas et al. ${ }^{13}$ Furthermore, the effects of the intrinsic layer width on GaN APDs is investigated by Wang et al. ${ }^{14}$

In contrast, analytical modeling of the spectral response for HPTs is rather limited. Chand et al. ${ }^{15}$ formulated analytical expressions for responsivity, however the boundary conditions considered were highly implausible for efficient HPT operation and no experimental comparison was provided. Several other attempts at predicting SR have been either limited to relative or normalized response ${ }^{16,17}$ or they require prior knowledge of device electrical characteristics. ${ }^{18,19}$ In this work, we have formulated expressions for flux dependent collector current leading to responsivity for HPTs and a detailed analysis on $\mathrm{Al}_{0.3} \mathrm{Ga}_{0.7} \mathrm{As} / \mathrm{GaAs} N p n$ HPTs is provided.

\section{SPECTRAL RESPONSE MODEL}

The spectral response model developed is based on the formulation of semiconductor continuity and diffusion 
equations in the base, depleted collector and subcollector layers of phototransistor. Suitable boundary conditions at the junctions of active device layers have been considered for efficient device performance. Subtle variation of refractive index caused by the changes in incident photon energies along with the effect of doping on absorption coefficient at near bandgap wavelengths has been taken into account. $^{20,21}$ The schematic for the HPT structure is shown in Fig. 1.

The base surface of the device is illuminated (Fig. 1) and the photoabsorption occurring in the base-collector (BC) depletion region and within the diffusion lengths of minority carriers will contribute toward the photoresponse. ${ }^{15}$ The steady state continuity equations governing the distribution of minority carriers for low-injection state in the base and subcollector regions are given by Eqs. (1) and (2), respectively. These incorporate drift and diffusion mechanism of excess minority carriers along with the photogeneration and recombination effects

$$
\begin{aligned}
& D_{n} \frac{d^{2} n_{p}}{d x^{2}}-\frac{\left(n_{p}-n_{p o}\right)}{\tau_{n}}+\phi_{b} \alpha_{b} \exp \left(-\alpha_{b} x\right)=0, \\
& D_{p} \frac{d^{2} p_{n}}{d x^{2}}-\frac{\left(p_{n}-p_{n o}\right)}{\tau_{p}}+\phi_{c} \alpha_{c} \exp \left(-\alpha_{c} x\right)=0,
\end{aligned}
$$

where $D_{n}$ (or $D_{p}$ ) and $\tau_{\mathrm{n}}$ (or $\tau_{\mathrm{p}}$ ) are diffusion coefficient and lifetime for minority carrier electrons (or holes), respectively, in $p^{+}$-GaAs base (or $n$-GaAs subcollector). $n_{p}$ (or $p_{n}$ ) and $n_{p o}\left(\right.$ or $\left.p_{n o}\right)$ are the total and equilibrium electron density (or hole) contributions, respectively, in the base (or subcollector) region. $\alpha_{\mathrm{b}}$ and $\alpha_{\mathrm{c}}$ are the optical absorption coefficients for the base and subcollector layers respectively. $\varphi_{\mathrm{b}}$ and $\varphi_{\mathrm{c}}$ are the values of incident flux density at the emitterbase $(x=0)$ and collector-subcollector $\left(x=w_{b}+w_{\text {dep }}\right)$ junctions, respectively. The boundary conditions taken at the emitter-base (E-B) edge has been written as:

$$
n_{p}(x=0)=n_{p}(0)=\frac{\phi_{O}\left(1-R_{f}\right)}{z(\alpha) s_{n}}=\frac{\phi_{b}}{z(\alpha) s_{n}}
$$

where $\varphi_{\mathrm{O}}$ is total incident flux density, $R_{f}$ is the Fresnel reflection coefficient and $s_{n}$ is the surface recombination velocity of electrons in $p^{+}$-GaAs base.

The surface recombination will be higher at lower wavelengths as more carriers are generated near the surface. This phenomenon is modeled by a wavelength dependent surface recombination parameter $z(\alpha)$. The excess minority carriers at the E-B junction are dependent on the input flux as no electrical contact is provided at the base terminal. Unity generation is taken for input flux which is limited by surface recombination of the photogenerated carriers. Optical flux is exponentially absorbed in the base region and the excess minority electron contribution at $x=w_{b}$, can be modeled by Eq. (4) which also involves the equilibrium electron contribution.

$$
\begin{aligned}
n_{p}\left(x=w_{b}\right) & =n_{p}\left(w_{b}\right)=\frac{\phi_{b}}{z(\alpha) s_{n}} \exp \left(-\alpha_{b} x_{b}\right)+n_{p o} \\
& =n_{p}(0) \exp \left(-\alpha_{b} x_{b}\right)+n_{p o} .
\end{aligned}
$$

The analytical resolution of Eq. (1), using the boundary conditions in Eqs. (3) and (4), enables one to determine the electron density in the base region. The total electron current, $I_{n}(\lambda)$, at the B-C junction, for a given wavelength radiation, can be evaluated using Fick's Law: ${ }^{22}$

$$
I_{n}(\lambda)=-\frac{q D_{n} A_{\mathrm{EB}}}{L_{n} \sinh \frac{w_{b}}{L_{n}}}\left\{\begin{array}{c}
C\left[\alpha_{b} L_{n} \sinh \left(\frac{w_{b}}{L_{n}}\right) \exp \left(-\alpha_{b} w_{b}\right)+\cosh \left(\frac{w_{b}}{L_{n}}\right) \exp \left(-\alpha_{b} w_{b}\right)-1\right]+ \\
n_{p}(x=0)\left[\cosh \left(\frac{w_{b}}{L_{n}}\right) \exp \left(-\alpha_{b} w_{b}\right)-1\right]+n_{p o}
\end{array}\right\}
$$

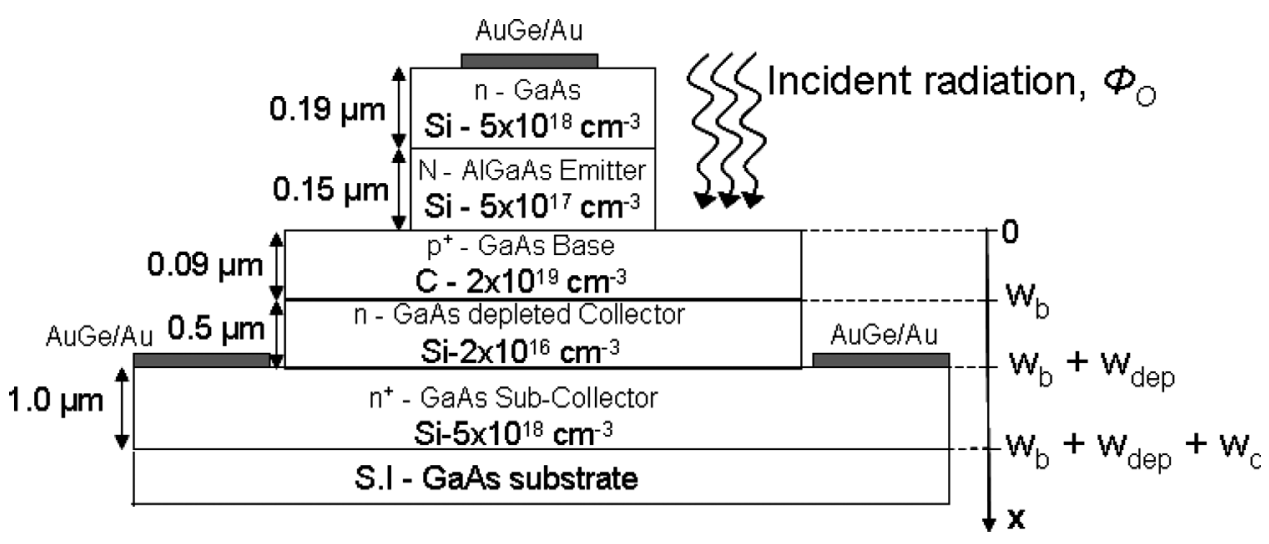

FIG. 1. Schematic device structure of the $\mathrm{Al}_{0.3} \mathrm{Ga}_{0.7} \mathrm{As} / \mathrm{GaAs}$ HPT showing the incident radiation on the base region. 
where $q$ is the charge of electron and $A_{\mathrm{EB}}$ is the E-B junction area. $L_{n}=D_{n} \tau_{\mathrm{n}}$ is the electron diffusion length in the base and $C$ is defined as
The collector current due to excess minority holes in the subcollector can similarly be modeled by following the procedure explained above and is written as

$$
C=\frac{\phi_{b} \alpha_{b} \tau_{n}}{\alpha_{b}^{2} L_{n}^{2}-1}
$$

$$
I_{p}(\lambda)=\frac{q D_{p} A_{C}}{L_{p} \sinh \left(\frac{w_{c}}{L_{p}}\right)}\left\{\begin{array}{c}
-C^{\prime}\left[1+\cosh \left(\frac{w_{c}}{L_{p}}\right) \exp \left(-\alpha_{c} w_{c}\right)+\sinh \left(\frac{w_{c}}{L_{p}}\right) \alpha_{c} L_{p} \exp \left(-\alpha_{c} w_{c}\right) \alpha_{c} L_{p}\right]+ \\
p_{n}\left(x=w_{b}+w_{d e p}\right)\left[1-\cosh \left(\frac{w_{c}}{L_{p}}\right) \exp \left(-\alpha_{c} w_{c}\right)\right]-p_{n o}
\end{array}\right\}
$$

where $C^{\prime}=\frac{\phi_{c} \alpha_{c} \tau_{p}}{\alpha_{c}^{2} L_{p}^{2}-1}$.

The optical flux absorption in the B-C depletion region gives rise to a photogenerated current $\left(I_{p h}\right)$ which can be modeled by Eq. (7). ${ }^{23}$

$$
I_{p h}(\lambda)=-q A_{C} \Phi_{d e p}\left[1-\exp \left(-\alpha_{d e p} w_{d e p}\right)\right]
$$

where $\Phi_{d e p}$ is the input flux density at the edge of B-C depletion region, $\alpha_{\text {dep }}$ is the absorption coefficient in the depletion region and $A_{C}$ is the area of the depleted collector. The fluxdependent collector current $\left(I_{c}\right)$ will be the sum of individual current contributions in the base $\left(I_{n}\right)$, B-C depletion $\left(I_{p h}\right)$ and collector $\left(I_{p}\right)$ regions.

$$
I_{C}(\lambda)=I_{n}(\lambda)+I_{p}(\lambda)+I_{p h}(\lambda)
$$

The responsivity, $\mathrm{R}(\lambda)$ is the ratio of photogenerated current to the input optical power and is given as ${ }^{24}$

$$
R(\lambda)=\frac{\lambda I_{C}(\lambda)}{h c \Phi_{O}\left(1-R_{f}\right)}
$$

where $h$ is Planks constant and $c$ is the velocity of light. This is a general expression for responsivity and can be used for any device materials provided one knows the details of device parameters.

\section{EXPERIMENETAL SETUP}

The $\mathrm{Al}_{0.3} \mathrm{Ga}_{0.7} \mathrm{As} / \mathrm{GaAs}$ Npn HPTs under investigation, with graded E-B junction, were grown by MOCVD and the devices were fabricated using standard mesa etching and photolithographic techniques. The $\mathrm{B}-\mathrm{C}$ and $\mathrm{B}-\mathrm{E}$ junction areas are $2.3 \times 10^{5} \mu \mathrm{m}^{2}$ and $2.3 \times 10^{4} \mu \mathrm{m}^{2}$, respectively. Transistors had DC current gains of about 23 at collector current of $2 \mathrm{~mA}$. The micrograph of fabricated device and the detailed device layer structure can be found elsewhere. ${ }^{25}$

The HPT wafers under investigation were set on a four DC-probe station. The laser diode (LD) is aligned to a multimode optical fiber ( $50 \mu \mathrm{m}$ core diameter) using collimating and focusing lenses. The optical radiation at the end of optical fiber is measured with an Anritsu optical power sensor MA 9802A, which is connected to an Anritsu optical power meter ML 092A.

The input signal to the laser is driven by an HP $8082 \mathrm{~A}$ pulse generator and the input current is modified by changing the DC offset voltage of the signal from the signal generator. The block diagram of the experimental setup is shown in Fig. 2. $L 1$ is a collimating lens and is used to obtain a parallel

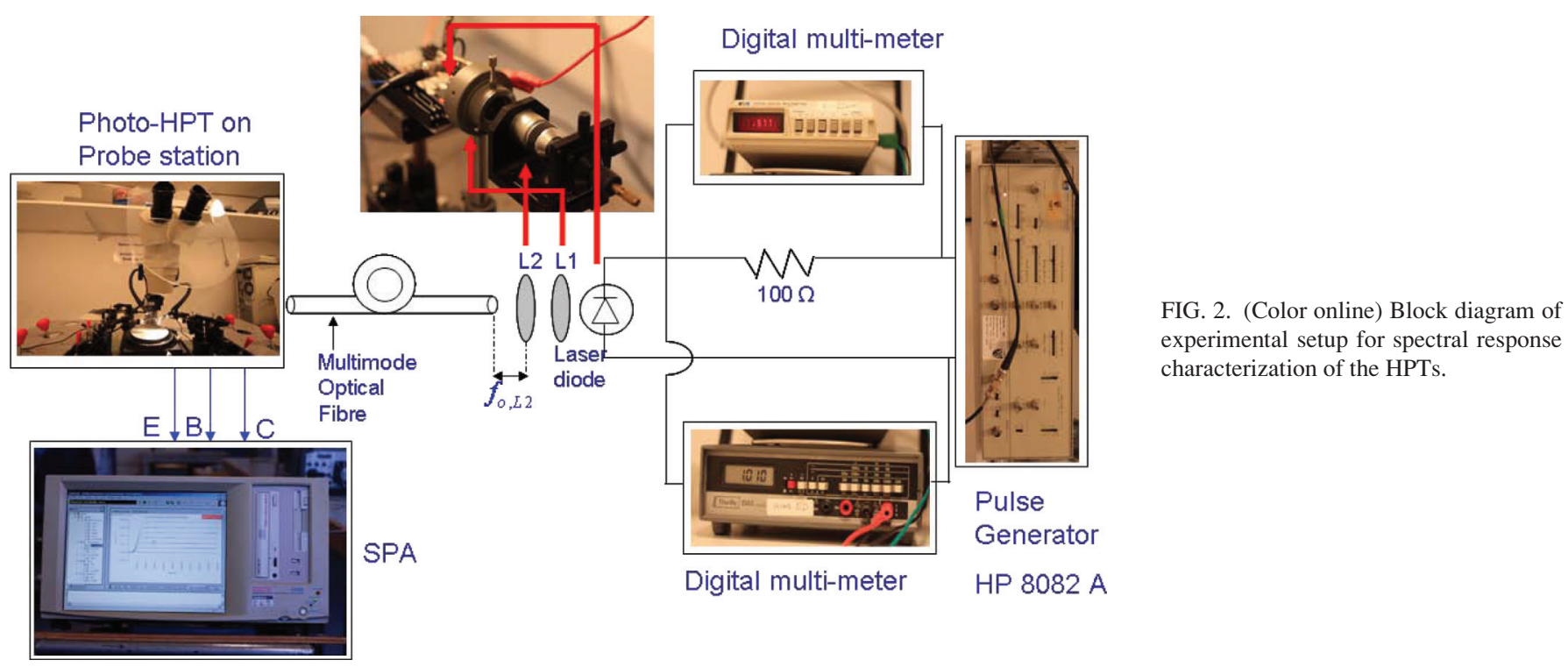




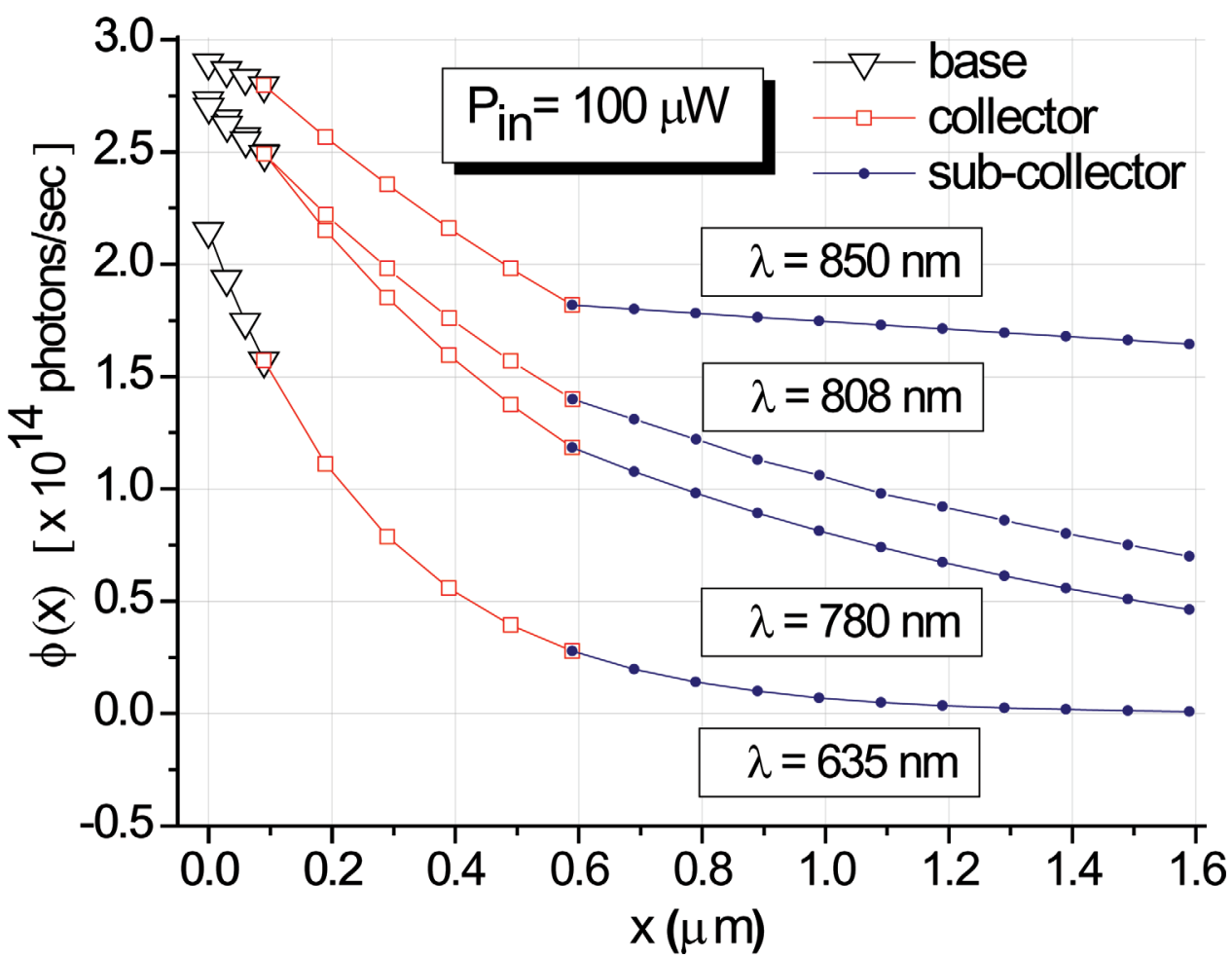

FIG. 3. (Color online) Optical flux absorption profile of the $\mathrm{Al}_{0.3} \mathrm{Ga}_{0.7} \mathrm{As} /$ GaAs HPT at various incident wavelengths for incident power of $100 \mu \mathrm{W}$. beam which is focused on the optical fiber through focusing lens $L 2$. The end of the optical fiber is placed at the focal length of $L 2\left(f_{o, L 2}\right)$ for optimal alignment. The semiconductor parameter analyzer (SPA), Keithley 4200, is capable of accurately measuring and graphically displaying DC characteristics of semiconductor devices.

\section{RESULTS AND DISCUSSION}

Optical flux absorption profiles for the understudy $\mathrm{Al}_{0.3} \mathrm{Ga}_{0.7} \mathrm{As} / \mathrm{GaAs} \mathrm{HPT}$ at $635 \mathrm{~nm}, 780,808$, and $850 \mathrm{~nm}$ radiations are given in Fig. 3. The different rate of absorption in the base $(0-0.09 \mu \mathrm{m})$, collector $(0.09-0.59 \mu \mathrm{m})$ and subcollector $(0.59-1.59 \mu \mathrm{m})$ is due to the variation of absorption coefficient in these layers. Doping concentration affects absorption coefficient for wavelengths closer to the bandgap of the material. Therefore, for GaAs (bandgap $=1.43 \mathrm{eV}$ ) the variation in absorption rate is most evident at $850 \mathrm{~nm}$. In contrast, the absorption coefficient is indifferent for the doping at $635 \mathrm{~nm}$ and this has been modeled by a single exponential. $^{21}$ At 780 and $808 \mathrm{~nm}$, the response tends to shift from a single exponential to layer dependent absorption as the variation in absorption coefficient is minute. A summary of absorption coefficients for GaAs, along with other material parameters, used for simulation is summarized in Table I.

Modeled collector current, using Eq. (8), along with the measured results at 780 and $850 \mathrm{~nm}$ are shown in Fig. 4. $I_{C}$ is higher for the same input power at $780 \mathrm{~nm}$ due to higher absorption coefficient which results in the generation of larger minority excess photocarriers for increased photoresponse. The spectral response of the devices is also shown in the inset with measured results for $635,780,808$, and 850 $\mathrm{nm}$. The base region of the large geometry phototransistor has been illuminated directly and so it is assumed that no input flux gets absorbed in the emitter region. Appreciable comparison with the measured results has been achieved as accurate boundary conditions have been considered and the effect of doping on the optical flux absorption profile is closely analyzed.

It should be noted that the surface recombination reduces the responsivity and requires careful modeling. The recombination due to misfit dislocations at the emitter-base heterostructure interface can be minimized by grading the heterojunction interface as used in this work. Despite the widely different surface preparation and measurement techniques, the surface recombination data for GaAs follows the same general trend of proportionality to bulk doping. ${ }^{29}$

The photocarriers, in close proximity to the surface are lost due to the higher recombination velocity. Therefore, an additional surface recombination parameter $z(\alpha)$ is required

TABLE I. Material parameters used for simulation (300 K).

\begin{tabular}{lll}
\hline \hline Parameter & Value (respectively) & Reference \\
\hline$D_{n}, D_{p}$ & $50,4\left[\mathrm{~cm}^{2} \mathrm{~s}^{-1}\right]$ & 21 \\
$\tau_{n}, \tau_{p}$ & $1 \times 10^{-9}, 1.2 \times 10^{-9}[\mathrm{~s}]$ & 26,27 \\
$L_{n}=\left(\tau_{n} D_{n}\right)^{1 / 2}$, & $2.2 \times 10^{-4}, 0.7 \times 10^{-4}[\mathrm{~cm}]$ & 22 \\
$L_{p}=\left(\tau_{p} D_{p}\right)^{1 / 2}$ & & \\
$s_{n}, s_{p}$ & $3 \times 10^{4}, \sim 10^{6}\left[\mathrm{~cm} \mathrm{~s}^{-1}\right]$ & 28,29 \\
$n_{p o}=n_{i}{ }^{2} / N_{A}$, & $2.2 \times 10^{-7}, 8.8 \times 10^{-7}\left[\mathrm{~cm}^{-3}\right]$ & 22 \\
$n_{p o}=n_{i}^{2} / N_{D}$ & & \\
$635 \mathrm{~nm}: \alpha_{\mathrm{b}}, \alpha_{\mathrm{dep}}, \alpha_{\mathrm{c}}$ & $3.4 \times 10^{4}, 3.4 \times 10^{4}, 3.4 \times 10^{4}\left[\mathrm{~cm}^{-1}\right]$ & 20,21 \\
$780 \mathrm{~nm}: \alpha_{\mathrm{b}}, \alpha_{\mathrm{dep}}, \alpha_{\mathrm{c}}$ & $1 \times 10^{4}, 1.4 \times 10^{4}, 0.9 \times 10^{4}\left[\mathrm{~cm}^{-1}\right]$ & $20,21,25$ \\
$808 \mathrm{~nm}: \alpha_{\mathrm{b}}, \alpha_{\mathrm{dep}}, \alpha_{\mathrm{c}}$ & $0.85 \times 10^{4}, 0.7 \times 10^{4}, 1.15 \times 10^{4}\left[\mathrm{~cm}^{-1}\right]$ & $20,21,25$ \\
$850 \mathrm{~nm}: \alpha_{\mathrm{b}}, \alpha_{\mathrm{dep}}, \alpha_{\mathrm{c}}$ & $0.6 \times 10^{4}, 0.9 \times 10^{4}, 0.1 \times 10^{4}\left[\mathrm{~cm}^{-1}\right]$ & 20,2125 \\
\hline \hline
\end{tabular}




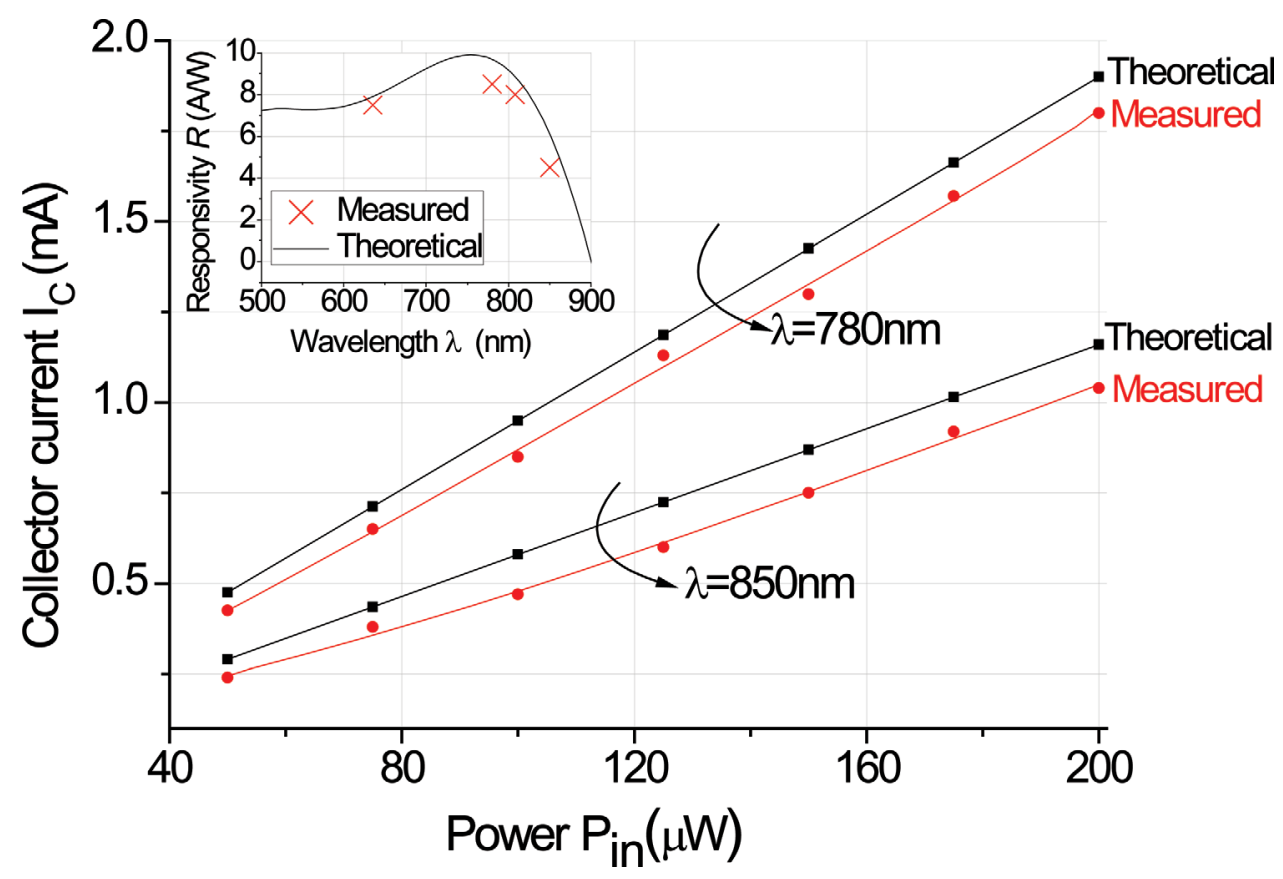

FIG. 4. (Color online) Measured and calculated spectral response of the $\mathrm{Al}_{0.3}$ $\mathrm{Ga}_{07} \mathrm{As} / \mathrm{GaAs} \mathrm{HPT}$. The inset (Reprinted with permission from H. A. Khan and A. A. Rezazadeh, IEEE Electron Device Letters, 30(11) 1158 (2009). Copyright (c) 2009. IEEE) shows a comparison between measured and theoretical responsivity of the phototransistor.

to model this effect. $z(\alpha)$ is a simulation parameter and the value of $z(\alpha)$ is higher for lower wavelengths as more photocarriers are generated near the surface. Remarkably, the variation of surface recombination parameter follows a near exponential decay with increasing wavelengths which is consistent with power absorption in semiconductors. The variation of $z(\alpha)$ with various incident wavelengths has been modeled in Fig. 5. Higher values of $z(\alpha)$ at lower wavelengths imply that a considerable amount of photo-generated carriers in the surface vicinity will be lost by recombination. Thus, these recombined carriers do not contribute to the photocurrent. It can also be inferred that the surface recombina- tion parameter for other material systems, such as lattice matched InP/InGaAs, will show similar decreasing trends with increasing wavelengths. However, the value of $\mathrm{z}(\alpha)$ will be smaller due to lower intrinsic surface and interface recombination in InP-based HPT devices as emitter and the base layer is lattice matched.

It can be observed from Fig. 6 that the responsivity shows a decreasing trend with the increase of base width. This data has been obtained by varying the base width in Eqs. (5), (6), and (7) and updating the values of the corresponding currents in Eq. (9) for three wavelengths under investigation. It is well known that the intrinsic current gain

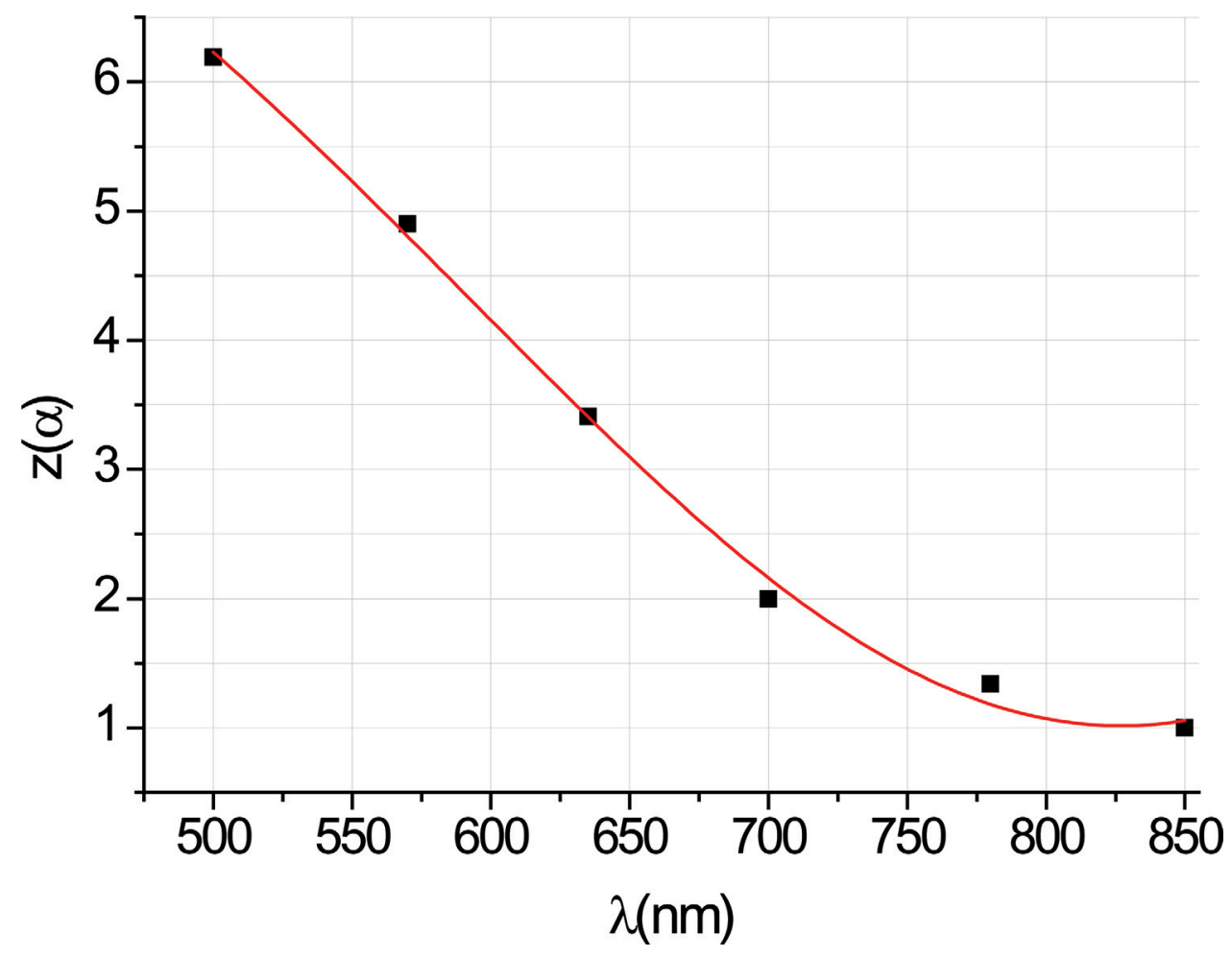

FIG. 5. (Color online) Simulated variation of surface recombination parameter $\mathrm{z}(\alpha)$ with incident wavelengths for the $\mathrm{Al}_{0.3} \mathrm{Ga}_{0.7} \mathrm{As} / \mathrm{GaAs}$ HPT. 


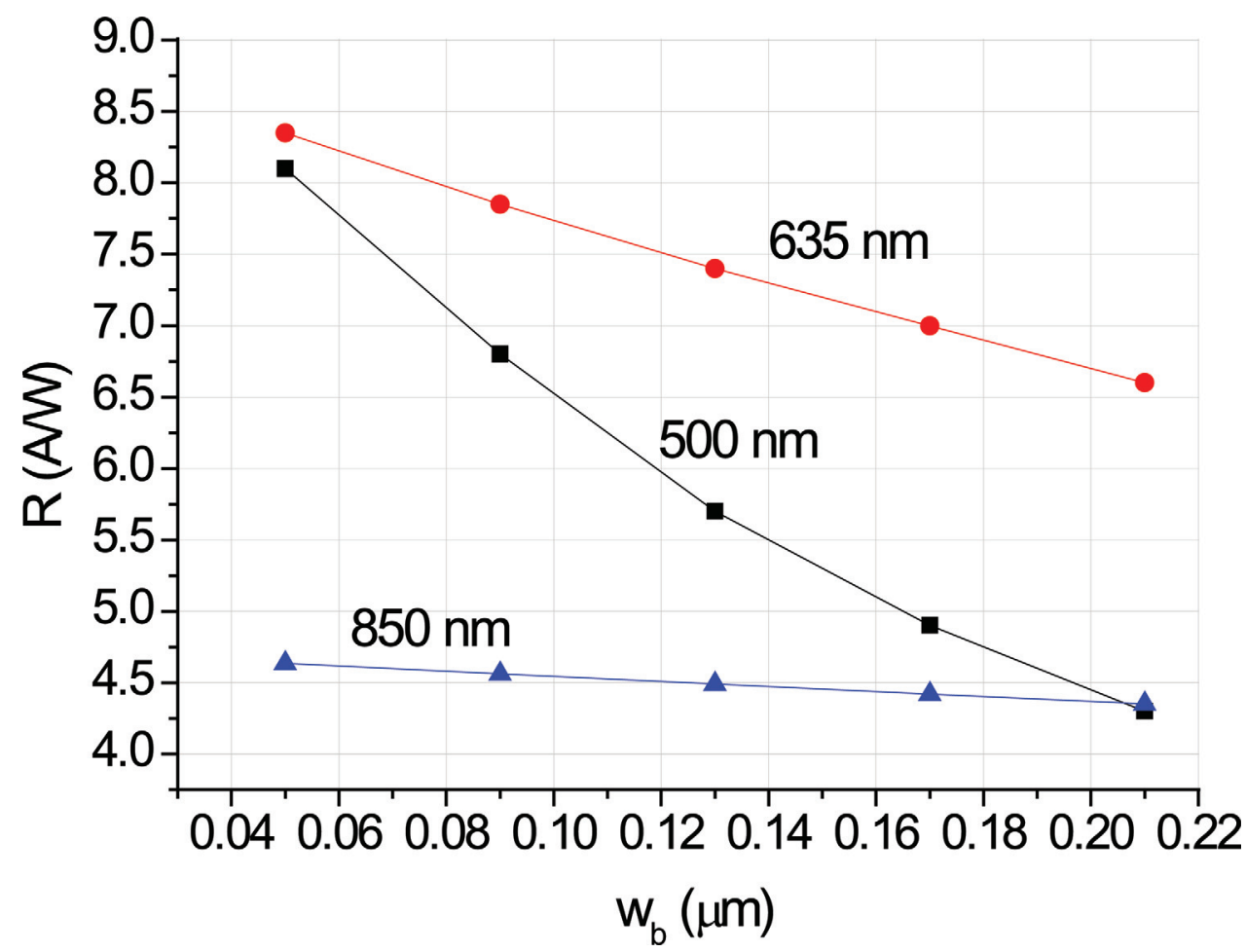

FIG. 6. (Color online) Simulated variation of responsivity with base width of the $\mathrm{Al}_{03} \mathrm{Ga}_{0.7} \mathrm{As} / \mathrm{GaAs} \mathrm{HPT}$ at various incident wavelengths.

of a transistor decreases with the increase in the base width due to increased recombination in the bulk region. However, different rates of decrease in the responsivity have been observed for three wavelengths in question, as seen in Fig. 6.

Increase in the base width is associated with an increase in bulk recombination as well as surface recombination because more carriers are being generated near the surface for high energy photons having smaller wavelengths. For the two wavelengths 635 and $500 \mathrm{~nm}$, relatively more photocarriers are generated near the surface for $500 \mathrm{~nm}$ as compared to $635 \mathrm{~nm}$ and as the surface recombination parameter is larger for $500 \mathrm{~nm}$, this result in a sharp drop in the responsivity at this wavelength. This trend could also be expected at $850 \mathrm{~nm}$, however the increase in recombination is compensated, largely, by the increased number of photocarriers generated in the base region.

The $\mathrm{Al}_{0.3} \mathrm{Ga}_{0.7} \mathrm{As} / \mathrm{GaAs}$ Npn HPT is optimized for detection at $850 \mathrm{~nm}$ as no or minute variation in the responsivity with changing base width is observed at this wavelength. At $635 \mathrm{~nm}$ and $500 \mathrm{~nm}$, responsivity increases with decreasing base width and vice versa. Depending on the application, the lower limit on the base width can be assessed using Monte Carlo simulation. ${ }^{30,31}$ This determines the boundary between the diffusion and ballistic modes for carrier transport. Responsivity generally decreases as recombination of minority electron in the $p$-type base will increase significantly with increasing base width. This analysis is particularly useful for photoreceivers based on double heterojunction phototransistors (DHPTs) as the absorbing width is limited for high frequency operations.

\section{CONCLUSION}

Closed form analytical expressions for the spectral response of HPTs presented in this paper provides an insight into spectral response modeling of HPTs. The model is based on the analysis of excess minority photocarrier generation, diffusion and recombination by applying semiconductor continuity equations. Accurate boundary conditions, for active regions of a phototransistor, have been described for in the theoretical model for efficient device performance. The analytical expressions and the critical analysis of several important parameters for HPTs presented here can be utilized for performance enhancement through device optimization for photoreceivers in optical networks and remote sensing applications employing integrated circuits.

\section{ACKNOWLEDGMENTS}

The authors would like to thank Tauseef Tauqeer and Asim Khan for valuable technical discussions. Hassan Khan would also like to express his gratitude to HEC Pakistan for the award of the studentship.

${ }^{1}$ H. Kamitsuna, Y. Matsuoka, S. Yamahata, and N. Shigekawa, IEEE Trans. Microwave Theory Techn. 49, 1921 (2001).

${ }^{2}$ R. F. Sridhara, S. M. Roenker, K.P. Pan, and N. Elliott, J. Lightwave Technol. 16, 1101 (1998).

${ }^{3}$ D. Ritter, B. Sheinman, V. Sidorov, S. Cohen, A. Gavrilov, Y. Vered, G. Zohar, and J. Lasri, Proceedings of International Topical Meeting on Microwave Photonics 5-8 Nov. 2002, Tokyo, Japan (IEEE, California, 2002).

${ }^{4}$ A. Leven, V. Houtsma, R. Kopf, Y. Baeyens, and Y.-K. Chen, Electron. Lett. 40, 833 (2004).

${ }^{5}$ S. M. Frimel and K. P. Roenker, J. Appl. Phys. 82, 3581 (1997).

${ }^{6}$ R. Decorby, R. Macdonald, M. Beaudoin, T. Pinnington, T. Tiedje, and F. Gouin, J. Electron. Mater. 26, L25 (1997).

${ }^{7}$ H. R. Chen, W. T. Chen, M. K. Hsu, S. W. Tan, and W. S. Lour, Semicond. Sci. Technol. 20, 548 (2005).

${ }^{8}$ A. Bouhdada, R. Marrakh, F. Vigue, and J.-P. Faurie, Reliab. 44, 223 (2004).

${ }^{9}$ M. A. Cappelletti, A. P. Cedola, E. L. Peltzer, and Y. Blanc, Semicond. Sci. Technol. 24, 105023 (2009). 
${ }^{10}$ M. A. Cappelletti, A. P. Cedola, E. L. Peltzer, and Y. Blanca, Semicond. Sci. Technol. 23, 025007 (2008).

${ }^{11}$ M. Hanzaz, A. Bouhdada, E. Monroy, E. Munoz, P. Gibart, and F. Omnes, Eur. Phys. J.: Appl. Phys. 11, 29 (2000).

${ }^{12}$ H. Zheng, K. Yong, T. Ying-Wen, L. Xue, and F. Jia-Xiong, Chin. Phys. 15, 1325 (2006).

${ }^{13}$ J. A. R. Gonzalez-Cuevas, T. F. Abedin, M. Nurul Elsayed-Ali, and E. Hani, Opt. Eng. 45, 044001 (2006).

${ }^{14}$ A. Bouhdada, R. Marrakh, F. Vigue, and J.-P. Faurie, Semicond. Sci. Technol. 24, 095006 (2009).

${ }^{15}$ N. Chand, P. A. Houston, and P. N. Robson, IEEE Trans. Electron Devices ED-32, 622 (1985).

${ }^{16}$ L. E. M. de Barros, Jr., A. Paolella, M.Y. Frankel, M.J. Romero, P.R. Herczfeld, and A. Madjar, IEEE Trans. Microwave Theory Tech. 45, 1368 (1997).

${ }^{17}$ S. A. Bashar and A. A. Rezazadeh, Proceedings of the 3rd IEEE International Workshop on High Performance Electron Devices for Microwave and Optoelectronic Applications, 27 Nov. 1995, London, UK, (IEEE, London, 1995).

${ }^{18}$ S. A. Bashar and A. A. Rezazadeh, IEE Proc.:-Optoelectron. 143, 89 (1996).

${ }^{19}$ H. A. Khan, A. A. Rezazadeh, and S. C. subramaniam, Proceedings of the European Microwave Integrate Conference, Amsterdam, 27-28 October 2008, (IEEE, Amsterdam, 2008), pp. 346-349.
${ }^{20}$ H. C. J. Casey, D. D. Sell, and K. W. Wecht, J. Appl. Phys. 46, 250 (1975).

${ }^{21}$ G. B. Lush, EMIS Data Review Series (Inspec Publications, 1990), pp. 513-528.

${ }^{22}$ S. M. Sze, Physics of Semiconductor Devices, 2nd ed. (Wiley, New York, 2001).

${ }^{23}$ K. Y. He Zheng, Tang Ying-Wen, Li Xue, and Fang Jia-Xiong, Chin. Phys. 15, 1325 (2006).

${ }^{24}$ H. A. Khan and A. A. Rezazadeh, IEEE Electron Device Lett. 30(11), 1158 (2009).

${ }^{25}$ H. A. Khan and A. A. Rezazadeh, IET Optoelectron. 4, 57 (2010).

${ }^{26}$ G. A. Acket, W. Nijman, and H. t. Lam, J. Appl. Phys. 45, 3033 (1974).

${ }^{27}$ C. J. Hwang, J. Appl. Phys. 42, 4408 (1971).

${ }^{28}$ J. R. M. Warner and B.L. Grung, Transistors: Fundamentals for the Integrated-Circuit Engineer (Wiley, New York, 1983).

${ }^{29}$ D. E. Aspnes, "Recombination at semiconductor surfaces and interfaces," Surface Science, vol. 132, pp. 406-421, 1983.

${ }^{30}$ R. J. Mills, D. C. Herbert, J. H. Jefferson, and A. B. Walker, Semiconductor Science and Technology, 8, 1719-1723 (1993).

${ }^{31}$ S. J. Woods, A. B. Walker, M. S. Stern, and D. W. E. Allsopp, IEEE International Topical Meeting on Microwave Photonics, Hungary, (IEEE, Budapest, 2003). 